\title{
microRNA-21 modulates cell proliferation and sensitivity to doxorubicin in bladder cancer cells
}

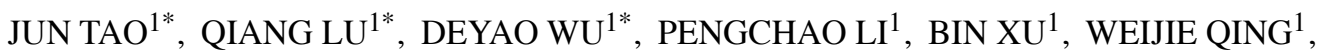 \\ MEILIN WANG ${ }^{2},{\text { ZHENGDONG } \text { ZHANG }^{2} \text { and WEI ZHANG }}^{1}$ \\ ${ }^{1}$ Department of Urology, The First Affiliated Hospital of Nanjing Medical University, 300 Guangzhou Road, \\ Nanjing 210029; ${ }^{2}$ Department of Molecular and Genetic Toxicology, School of Public Health, \\ Nanjing Medical University, 140 Hanzhong Road, Nanjing 210029, P.R. China
}

Received November 24, 2010; Accepted February 25, 2011

DOI: $10.3892 /$ or.2011.1245

\begin{abstract}
Transitional cell carcinomas (TCCs) of the urinary bladder are common malignancies with a high recurrence rate. Since microRNA-21 (miR-21) may contribute to tumorigenesis and chemoresistance in many cancer types, we aimed to investigate its efficacy in TCCs. The expression of miR-21 and its target PTEN was determined by real-time qRT-PCR and Western blotting, respectively in tumor tissues as well as adjacent non-tumor mucosa. The effect of miR-21 on cell proliferation and chemosensitivity to doxorubicin were measured using the MTT method. Cell apoptosis induced by doxorubicin was investigated using flow cytometry in the T24 cell line. BCL-2, AKT and pAKT were detected by Western blotting for analysis of potential mechanisms. miR-21 was significantly up-regulated in tumor tissues while PTEN was expressed in lower levels compared to non-tumor tissues. A negative correlation between expression of miR-21 and PTEN was established in vivo. Cell proliferation and chemoresistance to doxorubicin were promoted by overexpression of miR-21 in T24 cells. BCL-2 up-regulation could be achieved by miR-21 overexpression, which prevented T24 cells from apoptosis induced by doxorubicin. Furthermore, the miR-21 induced BCL-2 up-regulation could be cancelled by the PI3K inhibitor LY294002. These data verified the oncogenic role of miR-21 in TCCs and may usher in new therapeutic strategies in treating this disease.
\end{abstract}

Correspondence to: Dr Wei Zhang, Department of Urology, The First Affiliated Hospital of Nanjing Medical University, 300 Guangzhou Road, Nanjing 210029, P.R. China

E-mail: zhangwei_njmu@126.com

*Contributed equally

Key words: microRNA-21, bladder cancer, doxorubicin, chemosensitivity, PTEN

\section{Introduction}

Bladder cancer is one of the most common malignancies around the world, with about 70,980 new cases and 14,330 deaths in United States in 2008 (1). In China, it is the most common cancer in the urinary tract. Most of them are transitional cell carcinomas (TCCs), and approximately $70 \%$ tumors are confined to the mucosa or submucosa at the initial diagnosis. These non-muscle invasive tumors are typically treated with transurethral resection followed by intravesical therapy according to current guidelines. Doxorubicin and its derivants are commonly used as intravesical agents in treating bladder cancers. However, there are still 31-78\% patients suffering recurrence in 5 years. Moreover, about 20\% of recurred patients have disease progression (2). Recurrence usually occurs during or after intravesical chemotherapy, so chemoresistance may be one of the mechanisms of disease resurgence.

microRNAs (miRNAs) are short non-coding RNAs regulating gene expression post-transcriptionally by suppressing protein translation or accelerating degradation of mRNAs through non-stringent binding to mRNA 3 ' untranslated regions (3' UTR). miRNAs regulate expression of hundreds of target mRNAs simultaneously, thus control cellular processes, including proliferation, differentiation, apoptosis and tumorigenesis (3). There are over one thousand miRNAs registered in the database and among them miR-21 has been the best hit in a number of profiling experiments designed to detect deregulated miRNAs in many cancer types, including breast, colon, lung, pancreas, prostate, stomach $(4,5)$. Besides, miR-21 is also highly expressed in various cancer cell lines, suggesting its close relationship with carcinogenesis (6). Functional studies have also shown oncogenic role of miR-21. Overexpression of miR-21 often enhances cell proliferation, promotes cell cycle progression, increases anti-apoptotic activation, and boosts cell motility and invasion in various cancer cells while knockdown of miR-21 by chemically modified antisense oligonucleotides (ASO) usually renders opposite changes (7-10). Several recent reports have also shown that miR-21 modulates sensitivity to cytotoxic drugs and it is believed that higher miR-21 expression results in chemoresistance in many cancer types (11-14). These results put up a possible applica- 
tion of miR-21 as a therapeutic target. These observational phenomena are thought to be due to its suppression of several tumor suppressive genes, such as PTEN, PDCD4 or TPM1 $(9,15-17)$. However, the exact mechanisms underlying its wide effect are still unclear.

In TCCs, although several profiling studies have revealed miR-21 may participate in the tumorigenesis and most probably correlate to advanced diseases, functional study is still scarce $(18,19)$. In this study, we found miR-21 was highly expressed in advanced TCC tissues compared with its adjacent non-tumor tissues and negatively correlated to the protein level of PTEN as well. Furthermore, we showed the influence of miR-21 on cell proliferation in T24 cell line. Then, by calculating IC50 of doxorubicin we found that miR-21 could modulate the chemosensitivity of T24 cells to doxorubicin. We also demonstrated that these results could be, at least partly, attributed to modulating cellular anti-apoptotic BCL-2 expression, which manifested a dependence on PTEN mediated AKT activation.

\section{Materials and methods}

Tumor samples. All tumor samples were harvested during radical cystectomy and confirmed transitional cell carcinoma by pathological study post operatively. Adjacent non-tumor mucosa was also resected simultaneously, and half of it was sent to pathological inspection to rule out contamination of tumor. The collection and use of human tissues for investigational purpose was under the approval of our hospital's ethics committee. Tumor staging was determined by pTNM classification and tumor grading was according to WHO 2004 grading system.

Cell lines. Human bladder cancer cell line T24 was purchased from cell bank of Chinese Academy of Science (Shanghai, China). T24 cells were cultured in monolayer at $37^{\circ} \mathrm{C}$ and $5 \%$ $\mathrm{CO}_{2}$ with RPMI-1640 medium (Invitrogen) supplemented with $10 \%$ fetal bovine serum, 100 units/ml penicillin G (Sigma), $100 \mu \mathrm{g} / \mathrm{ml}$ streptomycin (Sigma). Cells were subcultured every 2 days using trypsin/EDTA solution (saline containing $0.05 \%$ trypsin, $0.01 \mathrm{M}$ sodium phosphate and $0.53 \mu \mathrm{M}$ EDTA, pH 7.4). PI3K inhibitor LY294002 was dissolved in DMSO (Beyotime Institute of Biotechnology, Jiangsu, China) and added to cells at the final concentration of $10 \mu \mathrm{M}$.

Real-time qRT-PCR. Total RNA was extracted from tissues using TRIzol reagent (Invitrogen). Real-time qRT-PCR for miR-21 was performed with taqman microRNA assay (Applied Biosystems) according to manufacturer's protocol. Briefly, a total $10 \mathrm{ng}$ RNA was used for the initial reverse transcription reaction using gene specific stem-loop RT primer available in the kit. Real-time PCR was performed on AB7300 thermo-recycler (Applied Biosystems) using miR-21 primer set and probes. Each sample was replicated three times with no RT and no template control included. Data were analyzed by comparing $\mathrm{Ct}$ values.

Transfection of the microRNA mimics or inhibitor. miR-21 mimics and its antisense inhibitor were designed according to mature miR-21 sequence. The sequence of miR-21 mimics was 5'-UAGCUUAUCAGACUGAUGUUGA-3' and the scrambled control was 5'-UUCUCCGAACGUGUCACGU TT-3'. The sequence of miR-21 antisense oligonucleotides with 2'O-Methyl modification was 5'-UCAACAU CAGUCUGAUAAGCUA-3', while the control sequence was 5'-CAGUACUUUUGUGUAGUACAA-3'. The sequences were synthesized by Genepharm Biotechnology (Shanghai, China) and verified by sequencing. Cells were transfected with $30 \mathrm{nM}$ mature miR-21 mimics, miR-21 antisense inhibitor, or controls using Lipofectamine 2000 transfection reagent (Invitrogen) at about $70 \%$ confluence according to the manufacturer's protocol.

Cell proliferation assay. Cells transfected with miR-21 mimics, inhibitor or controls were seeded in 96-well plates at a density of 3,000 cells per well. Cell proliferation was documented at 24, 48, 72 and $96 \mathrm{~h}$ after transfection. 3-(4, 5-dimethyl-2-thiazoyl)-2,5-diphenyl-2H-tetrazolium bromide (MTT) solution $(20 \mu \mathrm{l})$ were added into each well and incubated at $37^{\circ} \mathrm{C}$ for $4 \mathrm{~h}$. The plates were briefly centrifuged, and the purple colored precipitates of formazan were dissolved in $200 \mu \mathrm{l}$ DMSO. Absorbance was measured at $490 \mathrm{~nm}$ in an ELISA reader (Bio-Rad, Richmond, CA, USA). Six-well replication for every time point in each group was performed. Proliferation curves were drawn on the basis of mean absorbance at each time point.

Chemosensitivity assay. Forty-eight hours after transfection, cells were digested and plated in 96-well plates at the density of 3,000 cells per well. After an overnight incubation, the cells were treated with doxorubicin (Sigma) at various concentrations. Cell viability was measured after $48 \mathrm{~h}$ by MTT method as described. The suppression rate was calculated as follow: suppression rate $=\left(1-\mathrm{OD}_{\text {treated }} / \mathrm{OD}_{\text {control }}\right) \times 100 \%$. The doseresponse curve at different concentrations was charted to calculated the IC50 using a Probit regression model. Each concentration included 5 replicating wells and the experiments were performed 3 times independently to obtain a mean value of IC50.

Cell apoptosis assay. Forty-eight hours after transfection, T2 4 cells were seeded at a density of $1 \times 10^{6}$ cells per $10-\mathrm{mm}$ dish, and treated with doxorubicin at proper concentration for $24 \mathrm{~h}$. Then the cells were collected by brief trypsinization and washed twice in PBS. After incubation with $5 \mu 1$ of Annexin V-FITC and $10 \mu \mathrm{l}$ of propidium iodide (PI) at room temperature for 15 min in dark, cells were analyzed by flow cytometry (BD, NJ, USA). Apoptosis cells were recognized as high Annexin V fluorescence signal with low PI signal. The percentages of apoptotic cells were calculated by data from FACS analysis and the result was presented in the bar chart.

Western blot analysis. Primary antibodies used in this study including PTEN, AKT, phosphorylated AKT and BCL2 were products of Bioworld Technology (Louis Park, MN, USA). Antibodies against $\beta$-actin were purchased from Santa Cruz. Total protein of cells were prepared using RIPA lysis buffer. The concentration of protein was determined and a total $40-\mu \mathrm{g}$ protein was loaded in one lane in $12.5 \%$ SDS-polyacrylamide 
Table I. Clinical and pathological features of each sample as well as the relative expression of miR-21 and PTEN (mean \pm SD).

\begin{tabular}{|c|c|c|c|c|c|c|c|c|}
\hline \multirow[b]{2}{*}{ No. } & \multirow[b]{2}{*}{ Gender } & \multirow[b]{2}{*}{ Age } & \multirow[b]{2}{*}{ Stage } & \multirow[b]{2}{*}{ Grade } & \multicolumn{2}{|c|}{$\begin{array}{l}\text { Relative expression } \\
\text { of miR- } 21^{\mathrm{a}}\end{array}$} & \multicolumn{2}{|c|}{$\begin{array}{c}\text { Relative expression } \\
\text { of PTEN }\end{array}$} \\
\hline & & & & & $\mathrm{T}$ & $\mathrm{N}$ & $\mathrm{T}$ & $\mathrm{N}$ \\
\hline 1 & Male & 68 & T2N0M0 & High & $-5.56 \pm 0.08$ & $-2.65 \pm 0.37$ & $1.09 \pm 0.23$ & $0.53 \pm 0.16$ \\
\hline 2 & Male & 77 & T2N0M0 & High & $-7.49 \pm 0.35$ & $0.99 \pm 0.09$ & $1.30 \pm 0.17$ & $-1.29 \pm 0.27$ \\
\hline 3 & Female & 73 & T3N0M0 & High & $-11.41 \pm 0.70$ & $-1.9 \pm 0.28$ & $1.79 \pm 0.34$ & $1.03 \pm 0.35$ \\
\hline 4 & Male & 59 & T2N0M0 & High & $-5.04 \pm 0.28$ & $0.84 \pm 0.09$ & $1.25 \pm 0.16$ & $0.34 \pm 0.22$ \\
\hline 5 & Male & 48 & T2N0M0 & High & $-4.74 \pm 0.31$ & $2.08 \pm 0.14$ & $0.99 \pm 0.36$ & $-1.56 \pm 0.16$ \\
\hline 6 & Male & 71 & T3N1M0 & High & $-9.46 \pm 0.52$ & $1.99 \pm 0.05$ & $2.00 \pm 0.31$ & $-0.18 \pm 0.12$ \\
\hline 7 & Male & 66 & T2N0M0 & Low & $-7.02 \pm 0.51$ & $-0.4 \pm 0.13$ & $2.51 \pm 0.11$ & $0.35 \pm 0.09$ \\
\hline 8 & Male & 80 & T2N0M0 & High & $-8.00 \pm 0.65$ & $1.65 \pm 0.07$ & $1.24 \pm 0.28$ & $-0.39 \pm 0.14$ \\
\hline
\end{tabular}

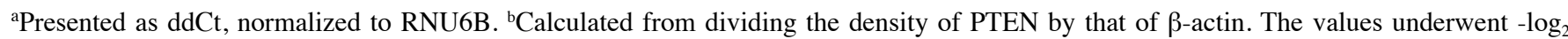
transformation. T, tumor; $\mathrm{N}$, adjacent non-tumor mucosa.

gels. After electrophoresis, the proteins were electro-transferred to polyvinylidene fluoride (PDVF) membranes. The membranes were blocked with $5 \%$ defatted milk and incubated with primary antibodies. The blots were then incubated with horseradish peroxidase-conjugated secondary antibodies (Santa Cruz). The blot was developed with ECL solution (Pierce, Rockford, IL, USA) and photographed by FluorChem imaging system (Alpha Innotech). The intensity of each spot was read and analyzed with AlphaEaseFC software.

Statistical analysis. Quantitative data are presented as mean $\pm \mathrm{SD}$, and compared using Student's t-test. Frequency data were presented directly and compared using the U test. IC50 was calculated from dose-response curve using a Probit regression model. All the analyses were performed with SPSS 12.0. P-value $<0.05$ was considered to be statistically significant.

\section{Results}

miR-21 is highly expressed in TCC tissues and negatively correlated to PTEN expression. We firstly determined the expression of miR-21 and its target gene PTEN in TCC tissues by real-time RT-PCR and Western blot analysis, respectively. For some technical reasons, this series of samples were collected during radical cystectomy and only represented advanced tumors (Table I). As depicted in Fig. 1, miR-21 was highly expressed in tumors compared to its adjacent non-tumor mucosa whereas PTEN was significantly lower in tumors, both $\mathrm{P}<0.05$. Correlation analysis showed a moderate negative correlation between expression of miR-21 and PTEN $(r=-0.86, P<0.01)$. These results confirm that miR-21 is a highly expressed miRNA in TCCs and may target tumor suppressor gene PTEN in vivo.

miR-21 promotes cell proliferation in T24 cells. Bladder cancer T24 cells have an aggressive phenotype and a moderate miR-21 level (19). To evaluate the biological impact of miR-21 on cell proliferation in T24 cells, we up- and down-regulated
miR-21 by transfecting synthetic miR-21 mimics or antisense inhibitor in T24 cells. As shown in Fig. 2A, cell proliferation was significantly promoted by up-regulation of miR-21 72 and $96 \mathrm{~h}$ after transfection comparing with negative control, while down-regulating miR-21 rendered a suspension of cell growth $48 \mathrm{~h}$ after transfection $(\mathrm{P}<0.05)$. These results indicate miR-21 may modulate cell growth and play an oncogenic role in bladder cancer cells.

miR-21 modulates chemosensitivity to doxorubicin in T24 cells. There is increasing evidence showing that anti-miR-21 treatment sensitizes chemotherapy in various cancer types. We tested whether miR-21 could modulate sensitivity of T24 cells to doxorubicin, a commonly used cell toxic drug in intravesical chemotherapy as well as systemic chemotherapy in advanced bladder cancer patients. Dose-response curves were charted for each group. Fig. 2B shows that the curve shifted inward for miR-21 mimics group compared with its control, which indicates desensitization to the drug. On the contrary, anti-miR-21 sensitized the cells to this agent as shown in Fig. 2C. Doxorubicin concentration of $50 \%$ inhibition of cell growth (IC50) was $1284.2 \pm 280.3$ $\mathrm{ng} / \mathrm{ml}$ for miR-21 mimics treated group and $689.4 \pm 58.3$ $\mathrm{ng} / \mathrm{ml}$ for negative control $(\mathrm{P}<0.05)$, whereas $369.7 \pm 65.8$ $\mathrm{ng} / \mathrm{ml}$ for anti-miR-21 group and $692.4 \pm 35.1 \mathrm{ng} / \mathrm{ml}$ for its control $(\mathrm{P}<0.05$, Fig. 2D). These results demonstrate that miR-21 could modulate chemosensitivity of T24 cells to doxorubicin.

miR-21 influences cell apoptosis induced by doxorubicin in T24 cells. Further, we performed flow cytometry to evaluate the influence of miR-21 on cell apoptosis induced by $500 \mathrm{ng} / \mathrm{ml}$ doxorubicin. As shown in Fig. 3, overexpression of miR-21 prevented cells from apoptosis at about 50\%, while down-regulation of miR-21 significantly increased cell death (both $\mathrm{P}<0.01$ ). This was consistent with the change of drug sensitivity and demonstrated that miR-21-induced chemosensitivity alteration was probably mediated by the cell apoptosis pathway. 
A

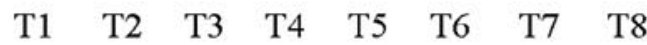

PTEN

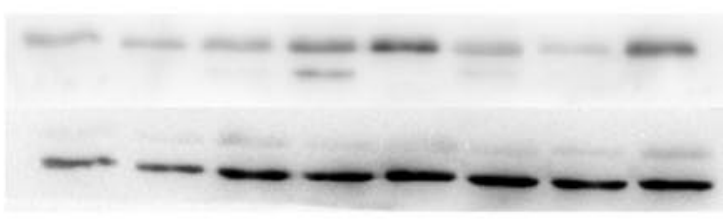

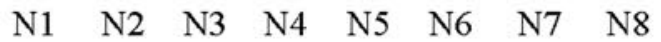

PTEN

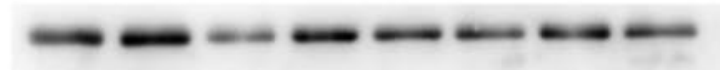

$\beta$-actin

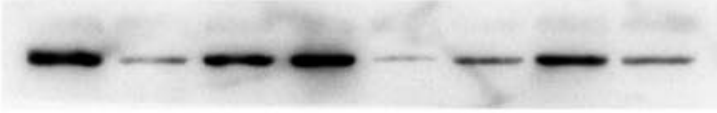

B

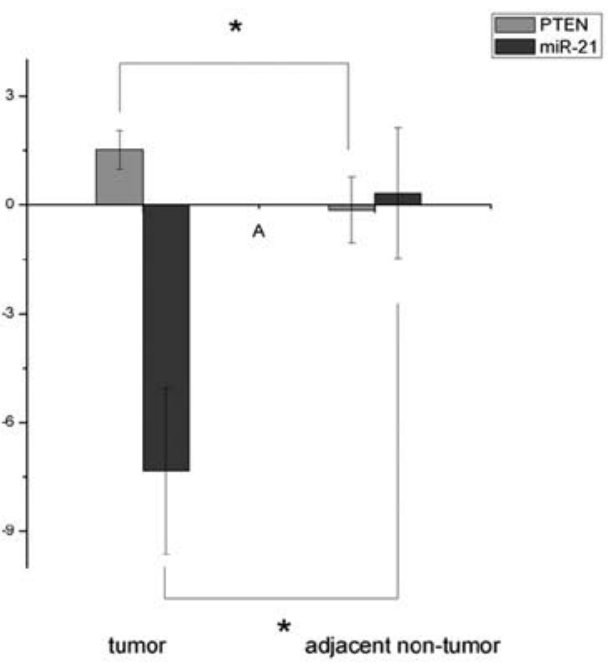

C

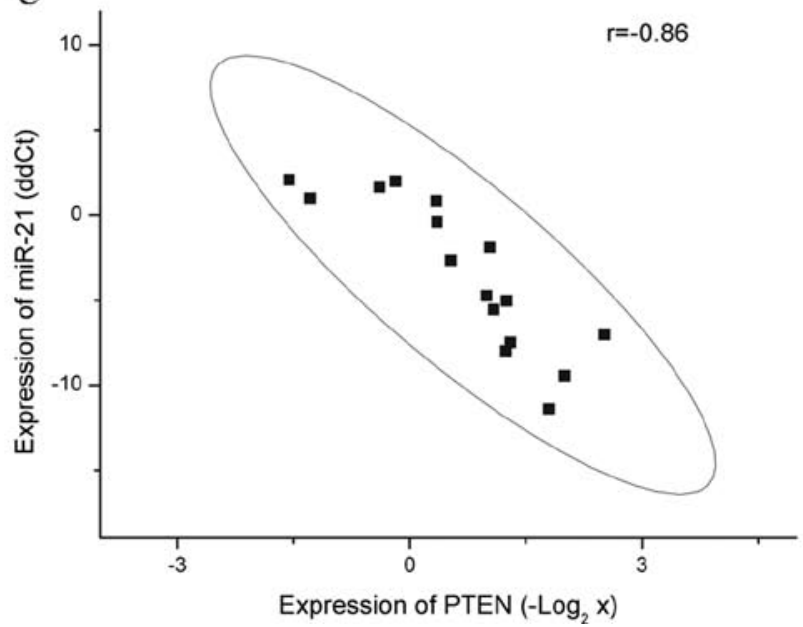

Figure 1. Expression of miR-21 and PTEN in TCC tissues. (A) Western blot analysis of PTEN in TCC tissues, $\beta$-actin as a loading control. T denotes tumor and $\mathrm{N}$ represents adjacent non-tumor mucosa. (B) Relative expression of miR-21 and PTEN after normalization. Expression data of PTEN were normalized to $\beta$-actin and underwent a $-\log _{2}$ transformation to get a logarithm form. Delta Ct between miR-21 and RNU6B is presented as logarithm form of relative level of miR-21. "P<0.05. (C) Pearson correlation analysis of expression of miR-21 and PTEN with 95\% confidence ellipse. Correlation coefficient was $-0.86, \mathrm{P}<0.01$.
miR-21 regulates $B C L-2$ expression in a PIBK/AKT-dependent manner. BCL-2 is considered a major pro-survival protein in challenged intracellular environment, which suppresses programmed cell death induced by various stress factors (20). We demonstrated in protein level that BCL-2 was up-regulated by transfection of miR-21 mimics and down-regulated by antimiR-21 treatment (Fig. 4A). Since BCL-2 has crosstalk with the PI3k-AKT pathway and miR-21 may target PTEN, a powerful endogenous suppressor of the PI3K pathway, we detected PTEN, AKT and pAKT for further analysis $(15,21,22)$. As expected, PTEN was under-expressed after miR-21 transfection and over-expressed after anti-miR-21 transfection. Furthermore, AKT, the key kinase in this pathway, was more phosphorylated to an active form after miR-21 overexpression (Fig. 4A and B). To investigate whether BCL-2 up-regulation induced by miR-21 is dependent on PI3k-AKT pathway, we inhibited PI3K by LY294002 pre-treatment, a well understood PIP3 inhibitor. As shown in Fig. 4C, pre-treatment of $10 \mu \mathrm{M}$ LY294002 apparently suppressed AKT phosphorylation but did not directly influence the expression of BCL-2 in protein level. However, BCL-2 up-regulation induced by miR-21 was dampened. These results indicate miR-21 could induce BCL-2 up-regulation probably in a PI3K-AKT-dependent manner.

\section{Discussion}

In the current study, we provided a functional investigation of miR-21 in bladder cancer in vitro, showing its critical role in cell proliferation, apoptosis as well as chemosensitivity. We also showed that the oncogenic role of miR-21 might be attributed to its suppression of PTEN in T24 cells.

miR-21 has been recognized as the most prominent miRNA implicated in carcinogenesis and is up-regulated in many cancer types. It is involved in promotion of tumor cell growth and proliferation, acceleration of cell cycle progression, suppression of cell apoptosis and induction of chemoresistance. Recent studies showed that the expression of miR-21 discriminated invasive phenotype from noninvasive one in TCCs $(18,19)$. Consistent with these studies, our results also demonstrated miR-21 was up-regulated and might play an oncogenic role in advanced bladder cancers, promoting cell proliferation in a bladder cancer cell line.

Cell growth is governed by multiple signal cascades including cell cycle control, growth factor pathways and protein synthesis. Among them, PI3k-AKT pathway may play a critical role. Various growth factor receptors, such as EGFR, utilize PI3K/AKT as signal transduction route (23). Furthermore, G $\beta$ L-Rictor-mTOR complex senses intracellular energy supply and transduces growth signal to the downstream AKT molecule, in this manner connecting extracellular stimulations with intracellular environments $(24,25)$. Phosphatase and tensin homolog (PTEN) is a $47-\mathrm{kDa}$ phosphatidylinositol-3,4,5-trisphosphate 3-phosphatase, containing a tensin-like domain and a catalytic domain similar to that of the dual specificity protein tyrosine phosphatases. This protein dephosphorylates phosphoinositide, negatively regulating intracellular level of PIP3 in cells and thus the AKT signaling pathway (26). PTEN is identified as a tumor 


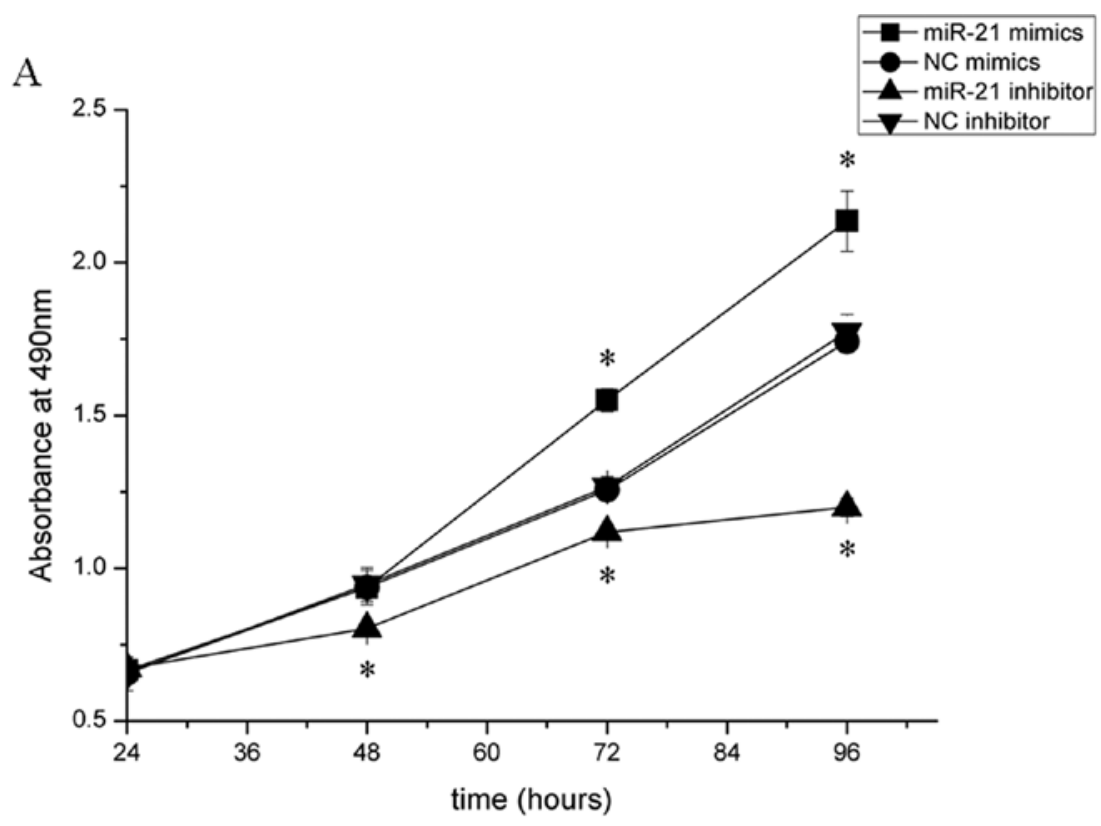

B

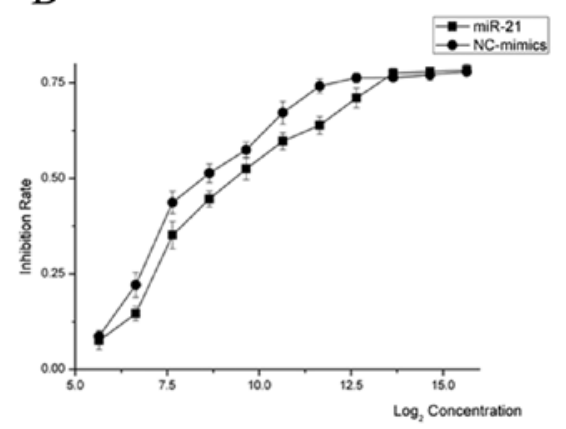

$\mathrm{C}$

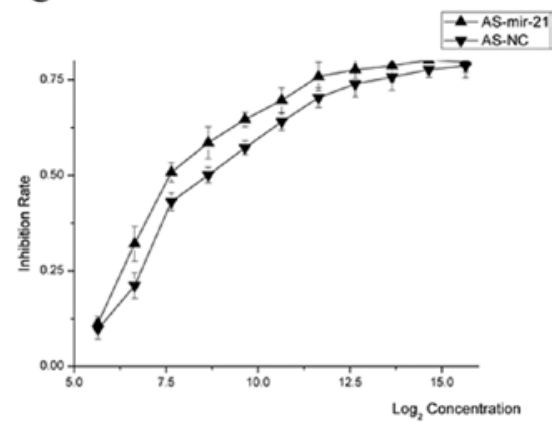

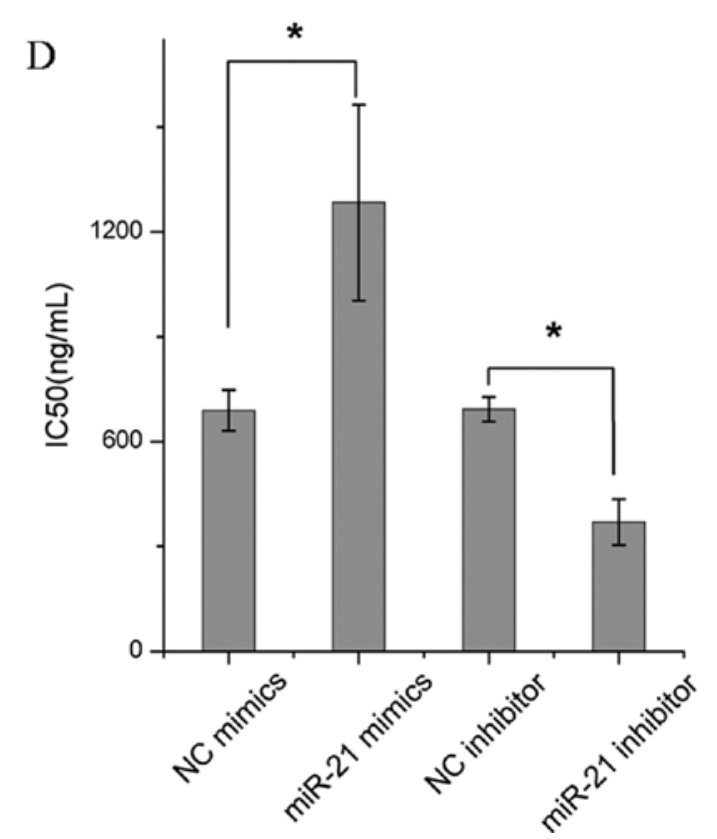

Figure 2. (A) Comparison of T24 cell proliferation after transfection with miR-21 mimics, antisense inhibitor and their negative controls respectively with MTT assay. Overexpression of miR-21 promoted cell proliferation $72 \mathrm{~h}$ after transfection while down-regulation of miR-21 affected cell growth $48 \mathrm{~h}$ later. ${ }^{*} \mathrm{P}<0.05$. (B) miR-21 mimics conferred chemoresistance to doxorubicin in T24 cells. The dose-response curve shifted downwards after miR-21 mimics transfection compared with its control. (C) miR-21 inhibitor sensitized cells to doxorubicin. The dose-response curve shifted upward when compared with negative control. (D) Comparison of IC50 of doxorubicin in each treatment group. IC50 was calculated from dose-response curve using a Probit regression model. Data are presented as the mean \pm SD from three independent experiments. " $\mathrm{P}<0.05$. 
A

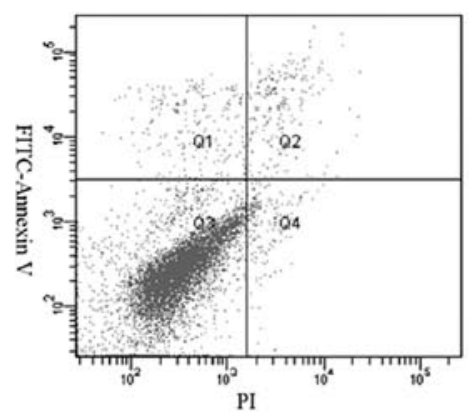

$\mathrm{C}$

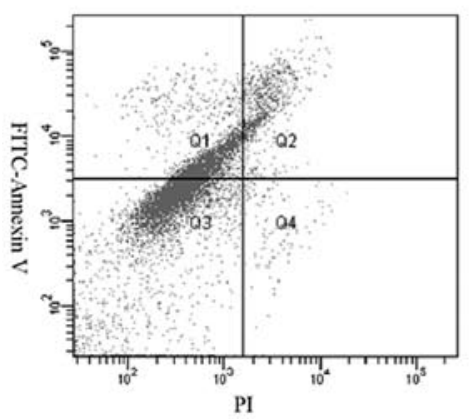

$\mathrm{E}$

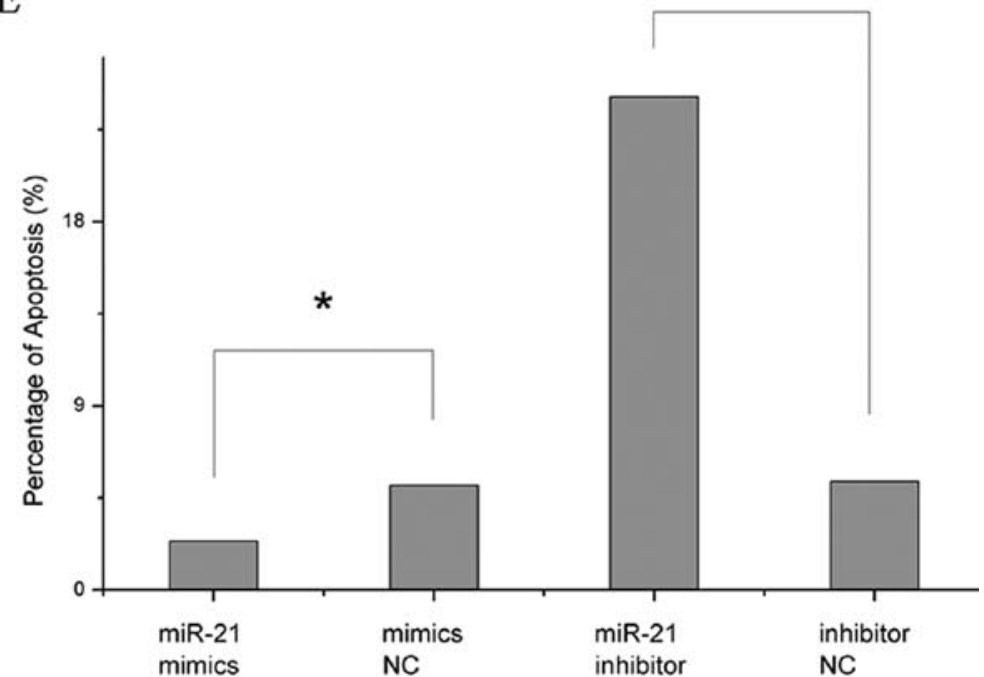

B

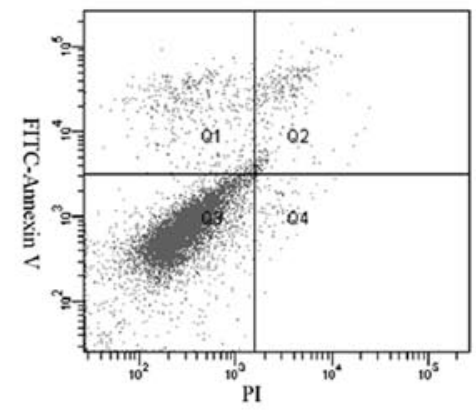

D

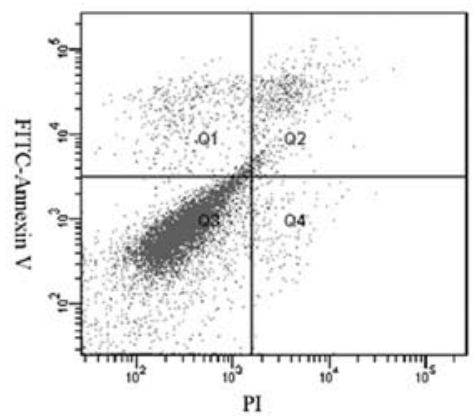

*

Figure 3. Flow cytometry assessment of cell proliferation induced by doxorubicin in each group. T24 cells were transfected with miR-21 mimics, antisense inhibitor and their negative controls, respectively. After 48-h incubation, doxorubicin was added to a final concentration of 500 ng/ml. After another 24-h incubation, cells were briefly trypsinized, washed and then collected for flow cytometry analysis. Annexin V and PI double staining was performed to reveal nuclear deformation in apoptotic cells. Cells showing high Annexin V signal with low PI signal were recognized as apoptosis (Q1). (A) T24 cells transfected with miR-21 mimics. (B) Cells transfected with negative control of mimics. (C) Cells transfected with miR-21 antisense inhibitor. (D) Cells transfected with negative control of inhibitor. (E) Compare of percentage of apoptotic cells in each group. Frequency data were compared using the $\mathrm{U}$ test. ${ }^{*} \mathrm{P}<0.01$.

suppressor and frequently mutated in a large number of cancers including bladder tumors (27). Besides genomic variation, transcriptional and post-transcriptional regulation may represent other avenues to PTEN absence. MicroRNAs stand for a novel mechanism mainly regulating gene expression post-transcriptionally by binding to mRNA 3 ' untranslated region. It has been reported that miR-21 targets PTEN and negatively regulates its expression, through which its oncogenic role was observed $(15,16,28,29)$. We found the 
A
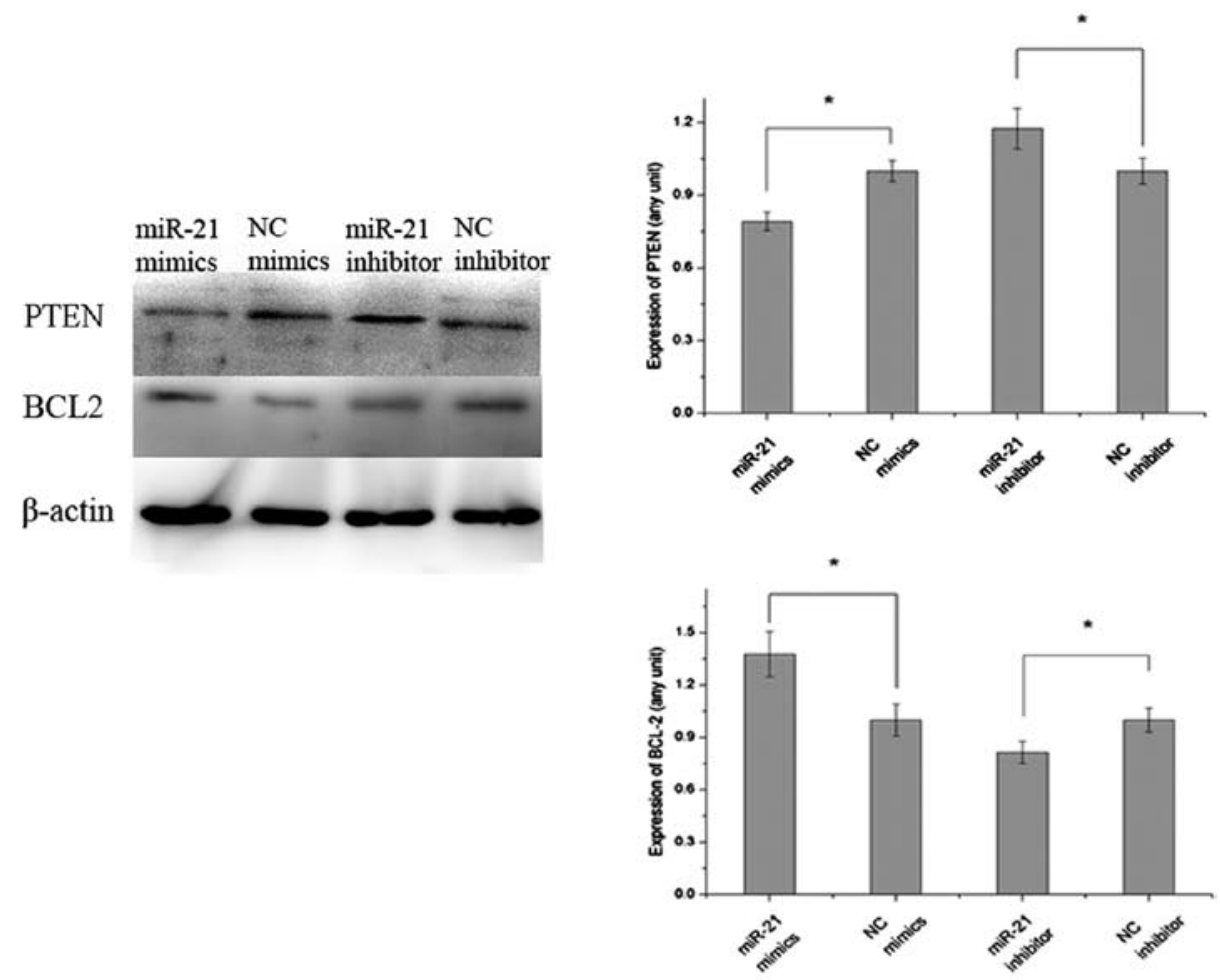

B

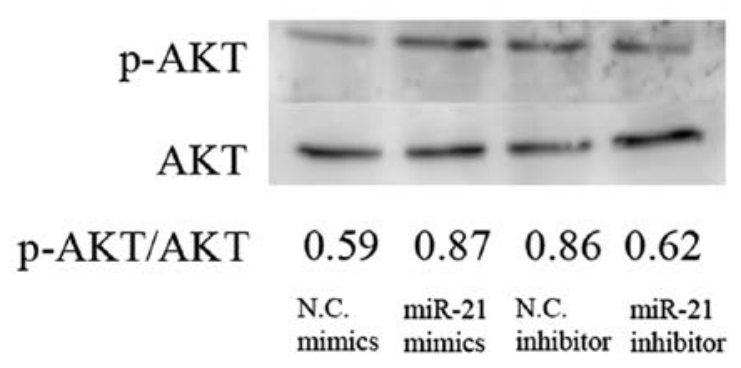

C

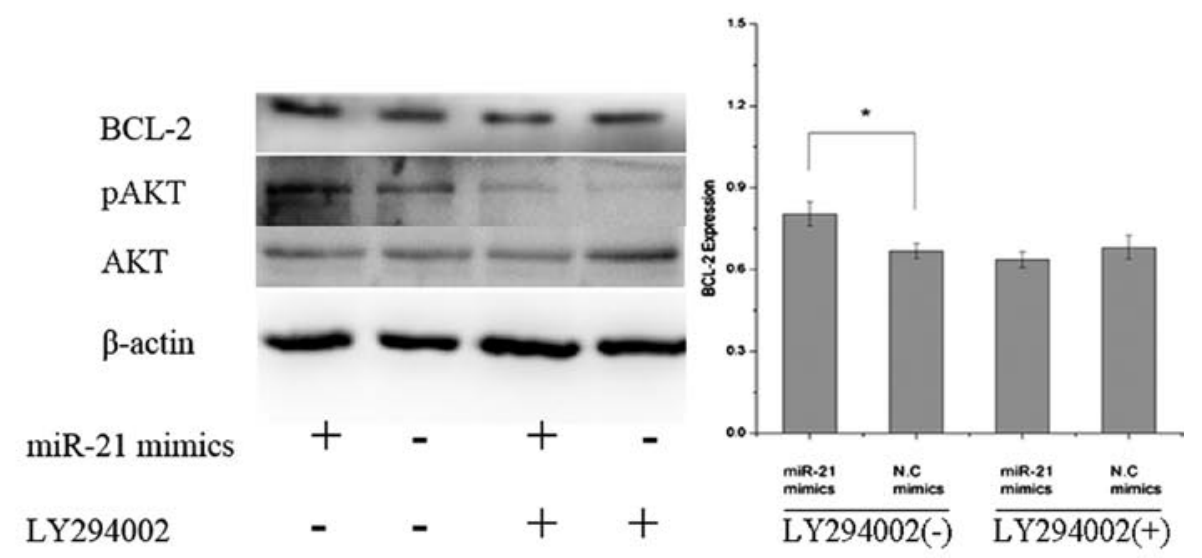

Figure 4. (A) Analysis of PTEN and BCL-2 expression in each treatment group by Western blot analysis. $\beta$-actin was used as a loading control. Data were normalized to $\beta$-actin and presented in a column chart. ${ }^{*} \mathrm{P}<0.05$. (B) Western blot analysis of p-AKT and AKT expression in each group. (C) miR-21 induced BCL-2 up-regulation can be cancelled by LY294002 treatment. LY294002 was added to a well at the final concentration of $10 \mu \mathrm{M}$ when changing culture medium after transfection and continued culture for $72 \mathrm{~h}$. LY294002 alone did not apparently influence BCL-2 expression albeit AKT phosphorylation was suppressed. However, BCL-2 up-regulation induced by miR-21 mimics was dramatically dampened.

elsewhere. Other targets such as PDCD4 (programmed cell death 4) and spry 2 may also contribute to the miR-21 induced cell proliferation enhancement. In this study, we only showed one aspect of a multi-faceted microRNA gene and we can not exclude other mechanisms which miR-21 may utilize. However, it seems that PTEN mediated the PI3k-AKT -dependent mechanism may play a role in oncogenic effect of miR-21 in TCCs. 
We also demonstrated for the first time that up-regulation of miR-21 could induce chemoresistance to doxorubicin in T24 cell line while down-regulation of miR-21 sensitized T24 cells to the drug. Doxorubicin is widely used as cytotoxic agent for intravesical and intravenous chemotherapy in superficial as well as muscle invasive bladder cancers. It is an anthracycline antibiotic which works by intercalating DNA so preventing DNA from being resealed, stops replication and eventually damages DNA structure. All these intra-nucleus events lead to cell growth arrest and apoptosis. Currently, drug resistance is the major reason for treatment failure. Several mechanisms underlie the development of chemo-resistance including enhanced drug pump out due to multi-drug resistance gene related pathway, increased DNA damage repair, and weakened cell apoptosis. miR-21 is thought to be a pro-survival microRNA showing antiapoptosis activity mainly due to its ability to induce BCL-2 expression, a powerful endogenous anti-apoptosis protein. It has been demonstrated that apoptosis induced by anti-miR-21 was due to the down-regulation of BCL-2 in the MCF-7 breast cancer cell line (10). In this study, we show that overexpression of miR-21 decreases T24 cell apoptosis induced by doxorubicin, inhibiting miR-21 increases cell death. Additionally, we revealed that miR-21 overexpression could induce BCL-2 expression and inhibition of miR-21 abrogated this effect. Furthermore, we found that miR-21 induced BCL-2 up-regulation could be cancelled by LY294002, showing its dependence on PI3K-AKT activation. However, PI3K inhibition itself seems not to be a direct influence on BCL-2 expression as revealed in this study as well as others (30). Thus, the exact molecular interactions by which miR-21 induces BCL-2 expression need further exploration.

Intravesical chemotherapy has long been recognized as a standard treatment for non-muscle invasive bladder cancer patients, with its effect inferior only to intravesical BCG irrigation (2). Considering the severe adverse reactions BCG induces, chemotherapy is probably the most acceptable choice for both physicians and patients. However, the recurrence rate is still unacceptable despite of intravesical therapies. Additionally, although many cytotoxic agents have been tested for treatment of this disease, none was clearly superior to others (2). This situation indicates that an intrinsic chemoresistance may play an important role. Our results showed that miR-21 may modulate cellular sensitivity to cytotoxic agent in vitro. Recent studies have shown the possibility of tansfering antisense oligonucleotides in treating bladder cancers (31). Bladder cancer, grown in an accessible lumen, may be a very good model to investigate this treatment strategy. Thus, our current results may guarantee further studies in vivo.

In conclusion, miR-21 is highly expressed in TCC tissues and promotes cell proliferation and chemoresistance in bladder cancer T24 cells probably through the PI3K-AKT pathway. These findings reveal the oncogenic role of miR-21 in TCCs and also provide a potential to develop novel strategies in treating TCCs.

\section{References}

1. Jemal A, Siegel R, Ward E, Hao Y, Xu J and Thun MJ: Cancer statistics, 2009. CA Cancer J Clin 59: 225-249, 2009.
2. Babjuk M, Oosterlinck W, Sylvester R, Kaasinen E, Bohle A and Palou-Redorta J: EAU guidelines on non-muscle-invasive urothelial carcinoma of the bladder. Eur Urol 54: 303-314, 2008.

3. Lee YS and Dutta A: MicroRNAs in cancer. Annu Rev Pathol 4: 199-227, 2009.

4. Griffiths-Jones S, Saini HK, van Dongen S and Enright AJ: miRBase: tools for microRNA genomics. Nucleic Acids Res 36: D154-D158, 2008.

5. Volinia S, Calin GA, Liu CG, et al: A microRNA expression signature of human solid tumors defines cancer gene targets. Proc Natl Acad Sci USA 103: 2257-2261, 2006.

6. Landgraf P, Rusu M, Sheridan R, et al: A mammalian microRNA expression atlas based on small RNA library sequencing. Cell 129: 1401-1414, 2007.

7. Gabriely G, Wurdinger T, Kesari S, et al: MicroRNA 21 promotes glioma invasion by targeting matrix metalloproteinase regulators. Mol Cell Biol 28: 5369-5380, 2008.

8. Asangani IA, Rasheed SA, Nikolova DA, et al: MicroRNA-21 (miR-21) post-transcriptionally downregulates tumor suppressor Pdcd 4 and stimulates invasion, intravasation and metastasis in colorectal cancer. Oncogene 27: 2128-2136, 2008.

9. Lu Z, Liu M, Stribinskis V, et al: MicroRNA-21 promotes cell transformation by targeting the programmed cell death 4 gene. Oncogene 27: 4373-4379, 2008.

10. Si ML, Zhu S, Wu H, Lu Z, Wu F and Mo YY: miR-21-mediated tumor growth. Oncogene 26: 2799-2803, 2007.

11. Ren Y,Zhou X, Mei M, et al: MicroRNA-21 inhibitor sensitizes human glioblastoma cells U251 (PTEN-mutant) and LN229 (PTEN-wild type) to taxol. BMC Cancer 10: 27, 2010.

12. Park JK, Lee EJ, Esau C and Schmittgen TD: Antisense inhibition of microRNA-21 or -221 arrests cell cycle, induces apoptosis, and sensitizes the effects of gemcitabine in pancreatic adenocarcinoma. Pancreas 38: e190-e199, 2009.

13. Moriyama T, Ohuchida K, Mizumoto K, et al: MicroRNA-21 modulates biological functions of pancreatic cancer cells including their proliferation, invasion, and chemoresistance. Mol Cancer Ther 8: 1067-1074, 2009.

14. Li Y, Li W, Yang Y, et al: MicroRNA-21 targets LRRFIP1 and contributes to VM-26 resistance in glioblastoma multiforme. Brain Res 1286: 13-18, 2009.

15. Meng F, Henson R, Wehbe-Janek H, Ghoshal K, Jacob ST and Patel T: MicroRNA-21 regulates expression of the PTEN tumor suppressor gene in human hepatocellular cancer. Gastroenterology 133: 647-658, 2007.

16. Vinciguerra M, Sgroi A, Veyrat-Durebex C, Rubbia-Brandt L, Buhler LH and Foti M: Unsaturated fatty acids inhibit the expression of tumor suppressor phosphatase and tensin homolog (PTEN) via microRNA-21 up-regulation in hepatocytes. Hepatology 49: 1176-1184, 2009.

17. Zhu S, Si ML, Wu H and Mo YY: MicroRNA-21 targets the tumor suppressor gene tropomyosin 1 (TPM1). J Biol Chem 282: 14328-14336, 2007.

18. Wszolek MF, Rieger-Christ KM, Kenney PA, et al: A MicroRNA expression profile defining the invasive bladder tumor phenotype. Urol Oncol: November 26, 2009 (Epub ahead of print).

19. Neely LA, Rieger-Christ KM, Neto BS, et al: A microRNA expression ratio defining the invasive phenotype in bladder tumors. Urol Oncol 28: 39-48, 2008.

20. Levine B, Sinha S and Kroemer G: Bcl-2 family members: dual regulators of apoptosis and autophagy. Autophagy 4: 600-606, 2008.

21. Tonic I, Yu WN, Park Y, Chen CC and Hay N: AKT activation emulates CHK1 inhibition and BCL2 overexpression and abrogates $\mathrm{G} 2$ cell cycle checkpoint by inhibiting BRCA1 foci. J Biol Chem 285: 23790-23798, 2010.

22. Zhang J, Han L, Zhang A, et al: AKT2 expression is associated with glioma malignant progression and required for cell survival and invasion. Oncol Rep 24: 65-72, 2010.

23. Navolanic PM, Steelman LS and McCubrey JA: EGFR family signaling and its association with breast cancer development and resistance to chemotherapy (Review). Int J Oncol 22: 237-252, 2003.

24. Zhang HH, Lipovsky AI, Dibble CC, Sahin M and Manning BD: S6K1 regulates GSK3 under conditions of mTOR-dependent feedback inhibition of Akt. Mol Cell 24: 185-197, 2006.

25. Astrinidis A and Henske EP: Tuberous sclerosis complex: linking growth and energy signaling pathways with human disease. Oncogene 24: 7475-7481, 2005. 
26. Maehama T and Dixon JE: The tumor suppressor, PTEN/ MMAC1, dephosphorylates the lipid second messenger, phosphatidylinositol 3,4,5-trisphosphate. J Biol Chem 273: 13375-13378, 1998.

27. Platt FM, Hurst CD, Taylor CF, Gregory WM, Harnden $P$ and Knowles MA: Spectrum of phosphatidylinositol 3-kinase pathway gene alterations in bladder cancer. Clin Cancer Res 15: 6008-6017, 2009.

28. Zhang JG, Wang JJ, Zhao F, Liu Q, Jiang K and Yang GH: MicroRNA-21 (miR-21) represses tumor suppressor PTEN and promotes growth and invasion in non-small cell lung cancer (NSCLC). Clin Chim Acta 411: 846-852, 2010.
29. Qi L, Bart J, Tan LP, et al: Expression of miR-21 and its targets (PTEN, PDCD4, TM1) in flat epithelial atypia of the breast in relation to ductal carcinoma in situ and invasive carcinoma. BMC Cancer 9: 163, 2009.

30. Zhuang J, Hawkins SF, Glenn MA, et al: Akt is activated in chronic lymphocytic leukemia cells and delivers a pro-survival signal: the therapeutic potential of Akt inhibition. Haematologica 95: 110-118, 2010.

31. Nogawa M, Yuasa T, Kimura S, et al: Intravesical administration of small interfering RNA targeting PLK-1 successfully prevents the growth of bladder cancer. J Clin Invest 115: 978-985, 2005. 